\title{
Knieprothesen werden zu früh implantiert
}

\author{
Der Kniegelenkersatz bei schwerer Arthrose ist der konservativen Therapie überlegen, \\ verursacht aber auch eher schwere Komplikationen - und erfolgt mehrheitlich zu früh.
}

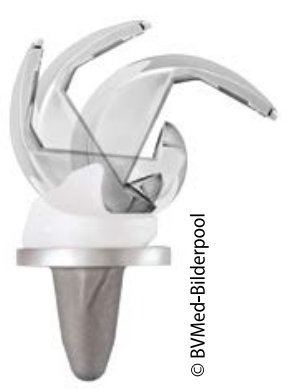

_ Für die Studie wurden 92 Patienten rekrutiert, bei denen die Indikation für eine unilaterale Kniegelenkprothese wegen mittelschwerer bis schwerer Arthrose bestand. Alle erhielten 12 Wochen lang Physiotherapie, Beratungen, Diätempfehlungen, individuell angefertigte Schuheinlagen und Analgetika. Die Hälfte $(\mathrm{n}=46)$ erhielt außerdem die Prothese. Zu Beginn und nach 12 Monaten wurden Schmerz, Beschwerden, tägliche Aktivität, Lebensqualität und körperliche Betätigung in einem kombinierten Score von 0-100 erfasst.

Nur 13 der 46 Patienten der Kontrollgruppe erhielten innerhalb von $12 \mathrm{Mo-}$ naten eine Prothese. Der Befindlichkeits-Score und die Funktionalität ver- besserten sich in der Operationsgruppe mit 32,5 signifikant stärker als in der Kontrollgruppe mit 16,0. Andererseits waren schwere Folgen (u.a. Venenthrombose, Steifigkeit mit Notwendigkeit zu Eingriffen unter Narkose, Infektion) und Nebenwirkungen allgemeiner Art (u.a. muskuloskelettal, gastrointestinal, dermal) bei operierten Patienten signifikant häufiger (24 vs. 6).

- Skou ST, Roos EM, Laursen MB et al. A Randomized, Controlled Trial of Total Knee Replacement. N Engl J Med. 2015;373:1597-606

\section{KOMMENTAR}

Kniegelenkprothesen haben sich rasch verbreitet, ohne dass der Nutzen im Vergleich zur konservativen Therapie jemals geprüft wurde. Diese methodisch zuverlässige Studie zeigt nun erstmals, dass die Prothese im ersten Jahr Schmerzen stärker lindert und Lebensqualität sowie Funktionalität deutlicher verbessert als die konservative Therapie. Dieser Vorteil wird erkauft durch eine vierfach erhöhte Zahl an schweren Komplikationen und Nebenwirkungen. Wichtig ist:

1. Alle fünf Kriterien des Scores haben sich auch unter der konservativen Therapie gebessert, mit der Prothese aber stärker.

2. Unter konservativer Therapie mussten bzw. wollten 3 von 4 Patienten sich im ersten Jahr nicht operieren lassen.

3. Ernsthafte Komplikationen traten nach der Operation viermal häufiger auf. Daraus folgt, dass die Mehrzahl der Patienen zu früh operiert wurde.

Prof. Dr. med. H. Holzgreve

\section{Hier steht eine Anzeige.}

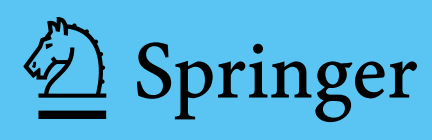

